\title{
Fuzzy Approach in Rail Track Degradation Prediction
}

\author{
Mostafa Karimpour $\mathbb{D}^{\mathrm{D}},{ }^{1}$ Lalith Hitihamillage, ${ }^{1}$ Najwa Elkhoury, \\ Sara Moridpour ${ }^{D},{ }^{1}$ and Reyhaneh Hesami ${ }^{2}$ \\ ${ }^{1}$ Civil and Infrastructure Engineering, RMIT University, Melbourne, Australia \\ ${ }^{2}$ Asset planning and Visualisation, Yarra Trams, Melbourne, Australia \\ Correspondence should be addressed to Mostafa Karimpour; mostafa.karimpour1@gmail.com
}

Received 9 October 2017; Revised 13 February 2018; Accepted 15 April 2018; Published 24 May 2018

Academic Editor: Lingyun Meng

Copyright (C) 2018 Mostafa Karimpour et al. This is an open access article distributed under the Creative Commons Attribution License, which permits unrestricted use, distribution, and reproduction in any medium, provided the original work is properly cited.

Rail transport authorities around the world have been facing a significant challenge when predicting rail infrastructure maintenance work. With the restrictions on financial support, the rail transport authorities are in pursuit of improved modern methods, which can provide a precise prediction of rail maintenance timeframe. The expectation from such a method is to develop models to minimise the human error that is strongly related to manual prediction. Such models will help rail transport authorities in understanding how the track degradation occurs at different conditions (e.g., rail type, rail profile) over time. They need a wellstructured technique to identify the precise time when rail tracks fail to minimise the maintenance cost/time. The rail track characteristics that have been collected over the years will be used in developing a degradation prediction model for rail tracks. Since these data have been collected in large volumes and the data collection is done both electronically and manually, it is possible to have some errors. Sometimes these errors make it impossible to use the data in prediction model development. An accurate model can play a key role in the estimation of the long-term behaviour of rail tracks. Accurate models can increase the efficiency of maintenance activities and decrease the cost of maintenance in long-term. In this research, a short review of rail track degradation prediction models has been discussed before estimating rail track degradation for the curves and straight sections of Melbourne tram track system using Adaptive Network-based Fuzzy Inference System (ANFIS) model. The results from the developed model show that it is capable of predicting the gauge values with $R^{2}$ of 0.6 and 0.78 for curves and straights, respectively.

\section{Introduction}

Modern transport organizations have shifted their focus from construction and expansion of the transport infrastructure towards how to intelligently maintaining them. This has taken place due to many reasons such as budget restrictions and running out of land space. Transport organizations currently focus on exploring the solutions for developing a maintenance management system that will help them accurately predict the time and location where maintenance should be carried out. This will assist transport infrastructure authorities in optimizing the cost and time of maintenance.

Different types of degradation prediction models have been presented in the literature, and these models have been mainly developed for the heavy rail system. Since there are differences in the structure and performance of heavy and light rail systems, it is impossible to use such degradation prediction models to predict the degradation of the light rail system. Consequently, it is needed to develop models which are capable of predicting the degradation of light rail tracks. This particular research will focus on developing a degradation prediction model for light rail network with the focus on tram network of Melbourne, Australia. The map of the current Melbourne tram network is shown in Figure 1.

The maintenance data of the Melbourne tram network has been collected through on-sight inspection and stocked in a nondigitized way for a long time. Traditionally, the rail maintenance used to be planned based on the experience of experts in the field. This procedure has changed since the introduction of new rail track inspection vehicles. These vehicles run through rail tracks and detect a large amount of data related to gauge and twist values from infrastructure condition. Based on this data, prediction models will be developed to predict the degradation of rail tracks 


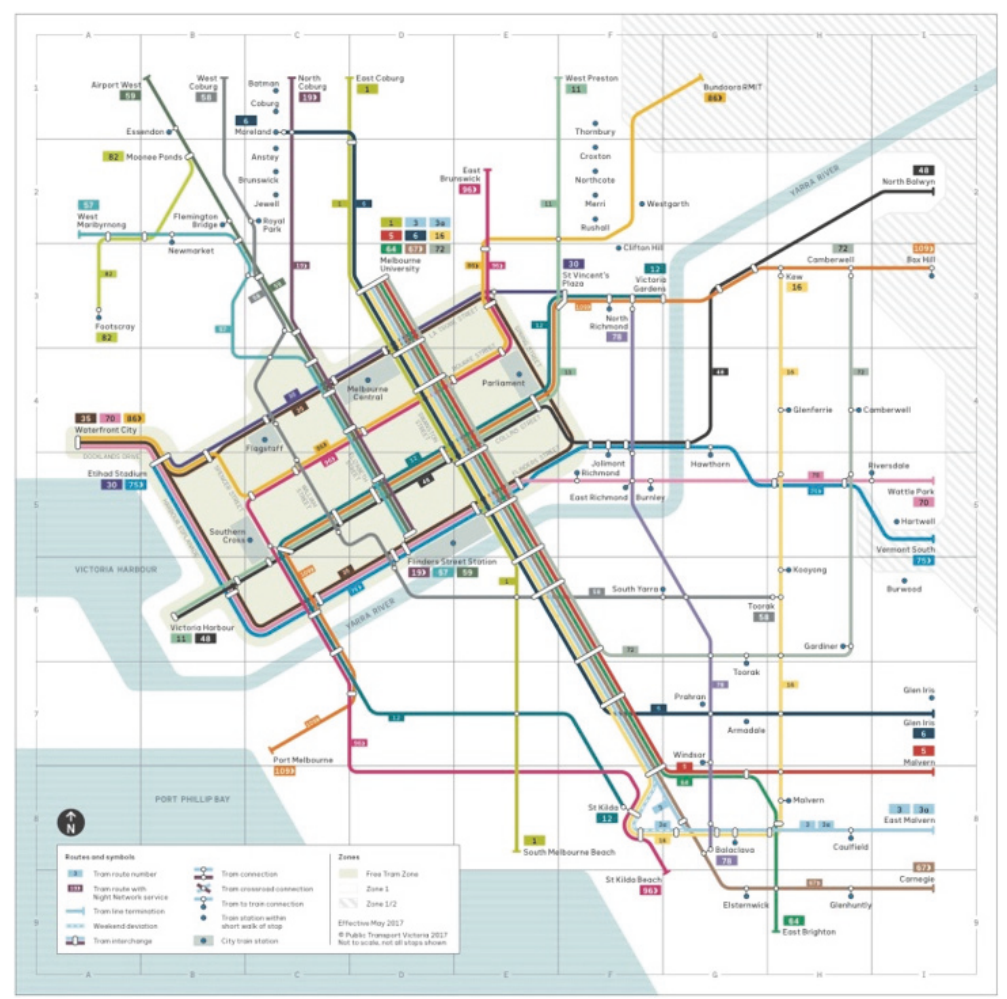

FIGURE 1: Melbourne tram network.

and estimate the maintenance procedures needed in the future.

In this paper, a review of the previous models on rail degradation prediction is presented. Afterwards, an ANFIS model is proposed to predict the Melbourne tram track degradation. Results from the models for both curves and straight sections are presented then. Finally, conclusions of this research and directions for further work on this topic are presented.

\section{Literature Review}

According to the literature, the models which have been used to predict rail track degradation could be categorized into three main categories including mechanistic, statistical, and artificial intelligence (AI) models. Out of these three main types, some of them are further categorized into subcategories as in Figure 2.

Previous studies on rail track degradation have represented some models that are capable of predicting degradation using a common set of parameters such as the age of the rail, axle load in Million Gross Tone (MGT), speed, and track curvature.

The mechanistic approach is the oldest model type in predicting the rail track degradation. Mechanistic models are based on the knowledge and understanding of the behaviour of the mechanical components. Mechanistic models involve establishing the mechanical properties either by theory or by testing. These types of models could mainly develop based on laboratory experiment data or collecting data from the field by observing the sections that will be used for modeling for a long period of time. Since such type of models has the capability of predicting rail track settlement and degradation with greater accuracy, according to the literature that is one of the key strengths of this type of models. So this is a type of model more suitable for utilizing in a situation where just one particular section of the track needed to be repaired. However, with this positive, there are few drawbacks that limit the possibility of using this type of models for predicting the future degradation and settlement values. The main drawback of this type of models is that it will not provide predictions with greater accuracy when you try to apply the model to the different sections of the rail network. The reason behind this is because the mechanical properties of the track and external factors they face may vary from place to place. Since it requires an extensive amount of data to develop a model small section of the rail track, developing a model for different sections of the network could be challenging and very time-consuming. Some of the models developed by the Japanese researchers [1-3] and German researchers [4] are among the most famous mechanistic models which have been pioneered, developing degradation prediction models based on mechanistic models around the globe.

The statistical models are based on the data collected from monitoring the track performances and the variables affecting such performances (e.g., traffic, rail type, and maintenance data). Those variables are used as inputs to develop a model which predicts track degradation. Statistical models provide the ability to deal with the considerably large amount of datasets when developing degradation models and they 


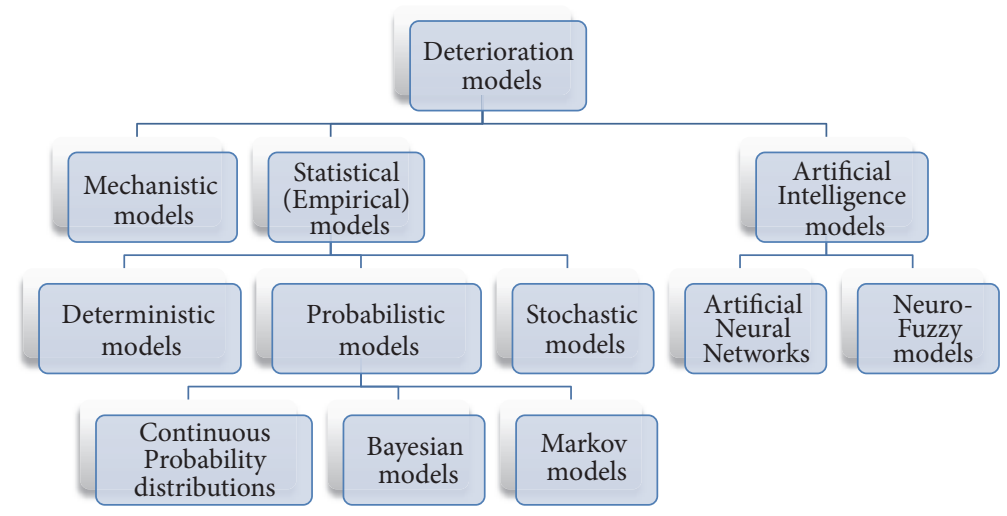

FIGURE 2: Rail track degradation prediction models.

provide more accurate results when applied to an entire rail network compared to a mechanistic model. However, it also has some downsides such as being able to create a large database which these types of models require due to the lack of availability of historical track data. Some of these statistical models could be further categorized into deterministic [5], probabilistic [6], and stochastic models [7].

The artificial intelligence models are a group of machine learning models which primarily could be categories into two groups' including artificial neural networks (ANNs) and neurofuzzy models. Artificial neural networks were originally inspired by the biological neural networks which are present in the human brain. These models are both simple computational devices which are highly interconnected, and in both models the connections between neurons determine the function of the network. Although ANNs are quite new to tram track degradation predictions, they have been extensively used in other fields of engineering [8]. ANNs are currently on the verge of becoming popular in predicting the degradation of railways due to their capability of providing prediction results with high accuracy $[9,10]$. They have also been utilized to evaluate the rail track condition [9]. The work of [9] proposed an ANN Model for track degradation prediction and used parameters such as Track Record Index (TRI) which defines the track quality. Moreover, traffic volume (i.e., light, heavy), speed, geographic location curves radius, and gradient are other factors used to predict the rail degradation [11]. Another study presented an artificial neural network model to predict the degradation of tram tracks using maintenance data in Melbourne railway system. Data was collected from Melbourne tram network from 2009 to 2013 , covering different types of segments of four routes such as straights, curves, $\mathrm{H}$-crossings, and crossovers [12]. Out of these segments, curves were the focus since they have a higher failure rate than some other segments [13, 14]; this section's data was utilized in case of light rail degradation modeling using a nonlinear method [15]. However, the results presented in previous researchs were far less accurate than what this paper has gained due to the higher nonlinear capability of ANFIS model.

In this research, at first the data are preprocessed, and all the outliers are eliminated. Following that, a fuzzy model is proposed due to the nonlinear and noisy nature of the data according to the data which were available on the Melbourne tram network. In the next stage, the model is validated on the test samples. The final year gauge values have been considered as the measured value for both training and testing experiments. Model validation/testing was done by comparing the final year gauge values as observed values and the gauge values for the final year estimated by the model.

Since gauge value represents the physical shape of railways, it can be considered as a factor which comprises various important features of rail degradation in its nature. Thus, rail degradation maintenance scheduling could be done by considering gauge degradation as a paramount factor. In this paper, gauge value degradation prediction modeling is done which could be a breakthrough into degradation hotspot detection which leads to lower amount of investment in both rail monitoring and preventive maintenance.

\section{Dataset}

Melbourne tram network is the world's largest urban operating tram network and covers $250 \mathrm{~km}$ of double tracks which includes 25 routes. There are 1700 stops across the network spread out with more than 400 level access stops. Seventyfive percent of the Melbourne's tram network operates on shared roads with other vehicles and manages to provide a service with a punctuality percentage around the high seventies to mid-eighties. To meet the current demands of the rapid growth of Melbourne city, its iconic tram network also needs to evolve accordingly. Along with the increase in Melbourne tram patronage, it has been understood that the expenses related to rail infrastructure maintenance have grown gradually and constantly.

Rail track degradation occurs due to many reasons. Rail vehicles travel at various speeds while carrying various loads. This will cause a wide range of stresses on the rail structure resulting in its decay. When it comes to the light rail tracks, the degradation of the track occurs due to few more other reasons such as the damage occurring by road sharing and weathering due to climate change. Since all these changes influence the decay and are embedded in gauge value, it is considered as the key value for the degradation. Gauge value is defined as the distance between the inner sides of two rails on a railway system. 


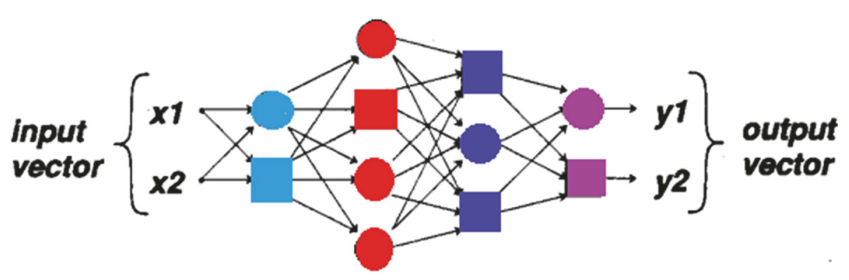

FIGURE 3: Simple adaptive network.

In this particular study, data are provided by Yarra Trams (which is the operator of the Melbourne tram network and is responsible authority for its maintenance). This data is utilized to develop a degradation model which will provide the ability to predict the future gauge values. The dataset which was provided by them for this research comprises different section types including curves, straight sections, $\mathrm{H}$-crossing, and crossovers. The gauge values have been collected by Yarra Trams for the whole tram network at different years. In addition, curve radius, annual tonnage in Million Gross Tone (MGT), track surface (asphalt and concrete surfaces), rail profile (the cross-sectional shape of a rail which is represented by kilogram per metre), rail type (grooved and T-shapes), rail support (or rail ties categorized into concrete and steel sleepers), location of routes, and track installation date have also been provided by Yarra Trams. These data were gathered over a period of 6 years from 2009 to 2015. Curve and straight sections where the two major groups of the track sections used in this study to analyze and develop the degradation prediction model since they represent the majority of the tram network.

\section{Model Development}

In this paper, ANFIS is used to estimate the gauge value for $t+1$ if the data for the past are available. The dataset is consisting of 3,860 different samples of gauge values between 2010 and 2015. Numerous sections of the railway had minor or major maintenance through these years. Thus, linear models are not able to consider the influence of different maintenance types over the years. Therefore, in this paper, an ANFIS model is proposed, and the results show the superiority of nonlinear models in the maintenance modeling. The ANFIS model presented in this paper consists of three important inputs including the two previous values of gauge (the previous two readings of the gauge from the past two years) and the MGT value. The dataset is divided into training and test set. $70 \%$ of the data is used for training purpose while the rest is used for testing.

An adaptive network can be considered as Figure 3 and is a feedforward multilayer network in which each node plays a particular role in the input with a set of parameters relating to the node [16]. Circle nodes have no parameter while the square nodes which are adaptive have different parameters that need to be estimated. If the network has $L$ layers and the $j$ th layer has $\#(j)$ nodes, the node in the $i$ th position of $j$ th layer can be written as

$$
O_{i}^{j}=O_{i}^{j}\left(O_{1}^{j-1}, \ldots, O_{\#(j-1)}^{j-1}, m, n, \ldots\right),
$$

where $m, n$, etc. are the parameters related to this node. $O_{i}^{j}$ represents the node output and the function. Considering a set of training data has $q$ entries the sum squared error could be measured as

$$
E_{q}=\sum_{m=1}^{\# L}\left(T_{m, q}-O_{m, q}^{L}\right)^{2},
$$

where $T_{m, q}$ is the $m$ th component of $q$ th target. The rate of error from the output node at $L, i$, can be derived from

$$
\frac{\partial E_{q}}{\partial O_{i, q}^{j}}=\sum_{m=1}^{\#(j+1)} \frac{\partial E_{q}}{\partial O_{m, q}^{j+1}} \frac{\partial O_{m, q}^{j+1}}{\partial O_{i, q}^{j}} .
$$

If $\theta$ is a parameter of the network $\partial E_{q} / \partial \theta$ can be written as

$$
\frac{\partial E_{q}}{\partial \theta}=\sum_{O^{*} \in S} \frac{\partial E_{q}}{\partial O^{*}} \frac{\partial O^{*}}{\partial \theta}
$$

$S$ is the node whose output depends on $\theta . \partial E / \partial \theta$ can be written as

$$
\frac{\partial E}{\partial \theta}=\sum_{q=1}^{q} \frac{\partial E_{q}}{\partial \theta}
$$

The update formula for $\theta$ can be written as

$$
\Delta \theta=-\gamma \frac{\partial E}{\partial \theta}
$$

in which $\gamma$ is the learning rate which can be defined as

$$
\gamma=\frac{j}{\sqrt{\sum_{\theta}(\partial E / \partial \theta)^{2}}}
$$

in which $j$ is the step size. The change in $j$ results in the convergence speed.

\section{Modeling and Results}

In this paper, 2,700 gauge observations were used to train the system. The input data are gauge values for year ${ }_{s-2}$, year $_{s-1}$, and MGT which are the most important factors in the one step ahead prediction for gauge values, and the output of the system is the gauge values for year ${ }_{s}$. The trained system antecedent membership functions are presented in Figure 4.

In this article firstly the datasets are randomly divided into training and testing datasets; following that ANFIS model is utilized using 5 membership functions for trio inputs. The number of membership functions and their shape are selected to have the Least Mean Squared Error (MSE) and higher $R^{2}$ in training datasets. These training datasets are then trained using ANFIS algorithm. Ultimately, the trained system is then tested on the test data and the observed gauge values and the predicted ones are compared as shown in Figure 5.

As shown in Figure 5, straight sections are responsible for more frequent and violent fluctuations in comparison to 

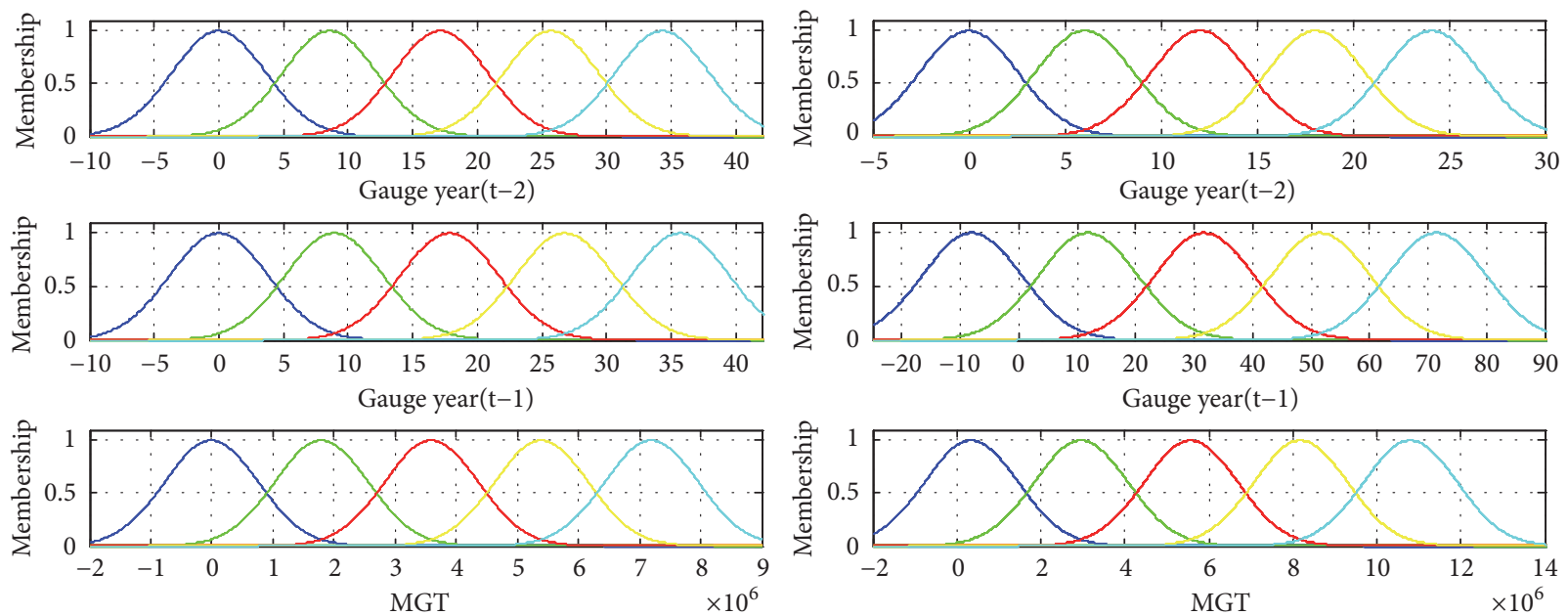

FIGURE 4: Membership function of the antecedents, i.e., the gauge values for year ${ }_{s-2}$ and year $r_{s-1}$ and MGT for curves and straight sections shown from left to right, respectively.

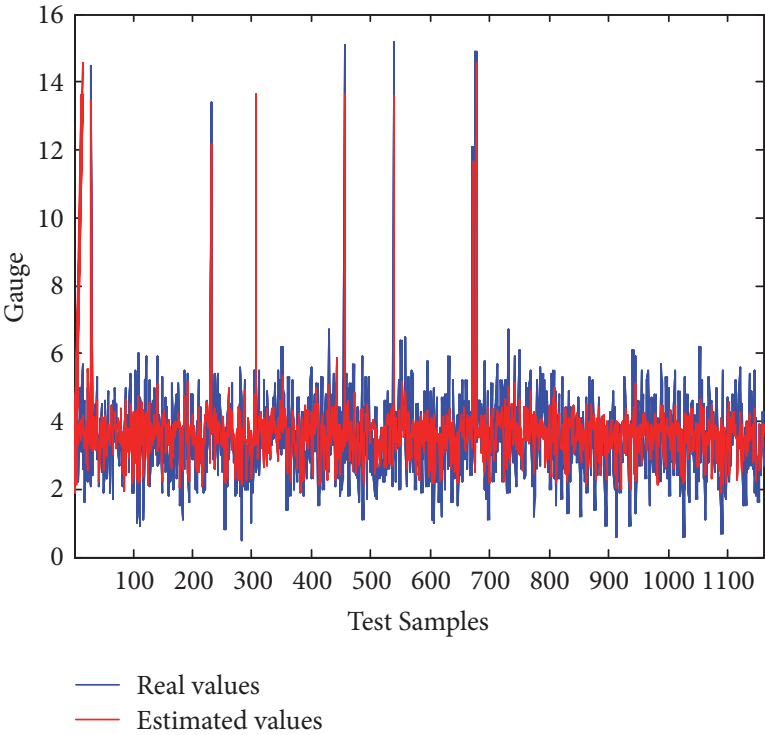

(a) Curve sections

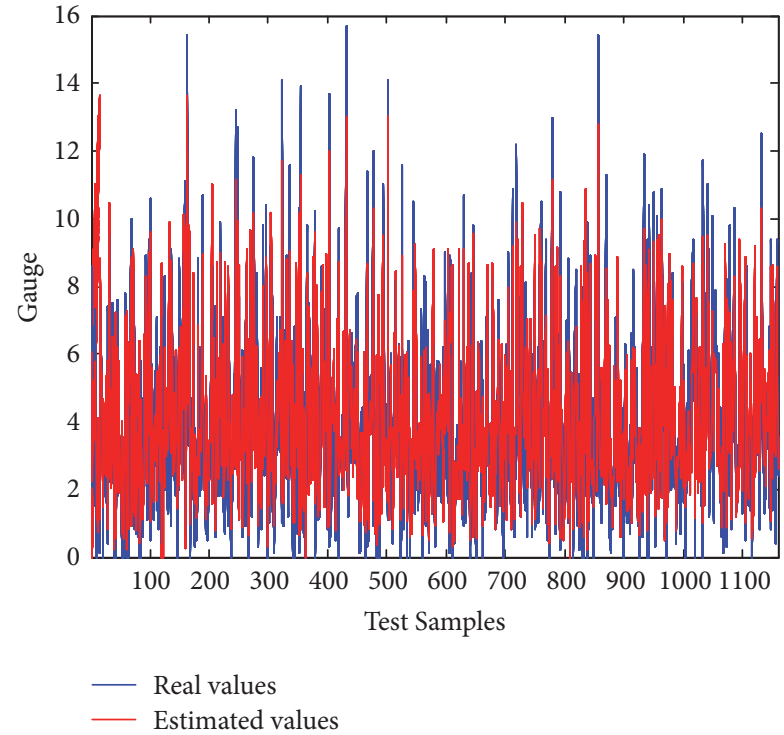

(b) Straight sections

FIGURE 5: The observed and predicted age values for the test data (30\% of the data).

curves. To unveil the key reason, it should be considered that switches and bridges which are more in danger of degradation are mostly quantified as straight sections. The spikes in both graphs reach a height of $14 \mathrm{~mm}$ in some samples. This occurrence of less frequent spikes could be justified by stating that it could be the result of noisy data in the curve sections. However, it would not be a valid argument when it comes to explaining the data of the straight section since the spikes in that graph are more frequent. This high decay in the gauge of the straight sections could be due to few reasons. It could be due to the subclasses of the straight sections eventually categorized as the straight sections. These sections tend to suffer greater deterioration in gauge than the other sections of the track. As a result, it will increase the overall gauge deterioration in the straight sections. To get more insight into the nature of the data in different sections Table 1 is presented.
TABLE 1: Mean and standard deviation for curves and straights. Real data is abbreviated as rd and estimated data is abbreviated as ed.

\begin{tabular}{lcc}
\hline & Curves & Straights \\
\hline$\sigma_{\text {rd }}$ & 1.3579 & 2.8207 \\
$\sigma_{\text {ed }}$ & 0.9766 & 2.4594 \\
$\mu_{\text {rd }}$ & 3.5320 & 3.9185 \\
$\mu_{\text {ed }}$ & 3.6160 & 4.0932 \\
\hline
\end{tabular}

Table 1 shows the mean and standard deviation of the two different sections.

Table 1 indicates the mean and the standard deviation for the observed and predicted gauge values at curves and straights, and it shows a massive difference in standard deviations between curve data and straight data in both 


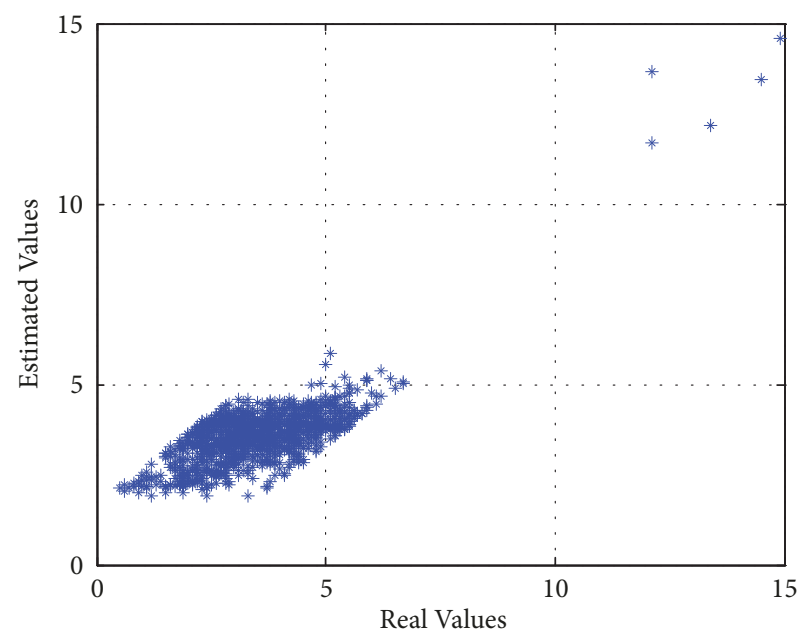

(a) Curve sections

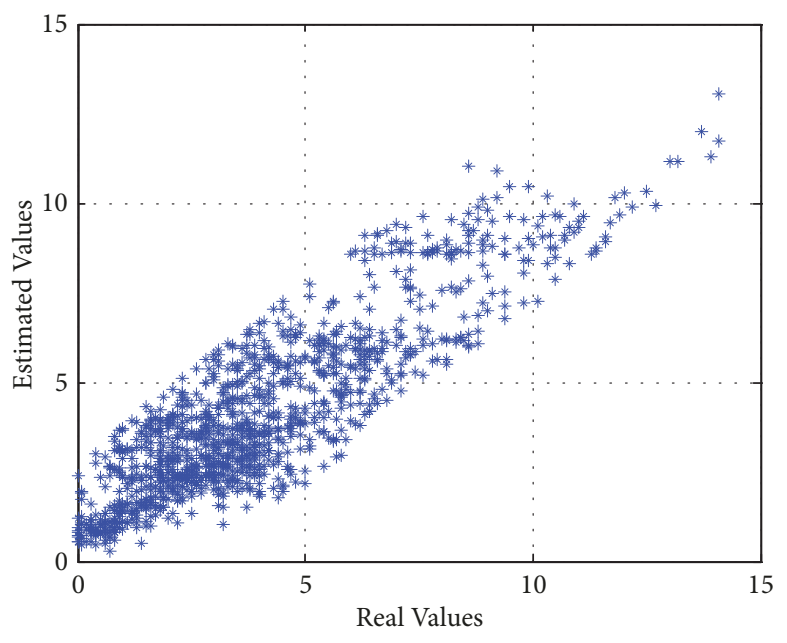

(b) Straight sections

FIGURE 6: The observed versus predicted gauge values.

observed and predicted values. The numbers demonstrate the nature of curves and straight sections. For instance, the values for the standard deviation in curves and straights which are 1.36 and 2.82, respectively, demonstrate that the deviation in the values of gauge value is higher in the straight section. Also, their mean values which are 3.53 and 3.92 for curves and straights demonstrate that the straight sections face higher gauge values. From both mean and standard deviation, it could be understood that higher gauge values are more common in the straight section which means this section type needs more common inspections due to the fact that straight section covers most frequent stop-starts and some other factors such as bridges which are enclosed by a straight section.

To show the accuracy of the model, observed gauge values versus the predicted gauge values are plotted in Figure 6. As demonstrated in this figure, the curve data may contain few outliers which could be seen in the top right corner of the curve graph. Through the graphical presentation provided in Figure 6, it is visible that the model has predicted those outliers accurately, given that they appear quite close to the regression line.

Moreover, Figure 6 depicts that the concentration of the gauge values lies within 0 to 5 in both graphs while on the straight sections there is a considerable spread in gauge values from 5 to 10 . This indicates a higher probability of degradation on the straight sections over curves.

$R^{2}$ which indicates the proportionate amount of variation in the response variable $y$ explained by the independent variables $X$ in the linear regression model is presented in (8). $R^{2}$ of the curve degradation prediction model is 0.60 while that of the straight model is around 0.78. By considering the above figures and the values of $R^{2}$, it is clear that the system can predict the gauge values with high accuracy considering the noisy nature of the data.

$$
R^{2}=\frac{\mathrm{SSR}}{\mathrm{SST}}=1-\frac{\mathrm{SSE}}{\mathrm{SST}}
$$

TABLE 2: Statistical parameters of model.

\begin{tabular}{lcc}
\hline Criteria & Curves & Straights \\
\hline$R$ square $\left(R^{2}\right)$ & 0.6001 & 0.7808 \\
MSE & 0.7350 & 1.7335 \\
Total Samples & 3860 & 3860 \\
Training Samples & 2700 & 2700 \\
Testing Samples & 1160 & 1160 \\
Number of inputs & 3 & 3 \\
\hline
\end{tabular}

where SSE is the sum of squared errors; SSR is the sum of squared regressions; SST is the squared total sum.

As Table 2 indicates, 3,860 observations have been used to train and test ANFIS models for predicting the gauge values for curve and straight sections. Despite the fact that the model for straight sections shows a better $R^{2}$ value, the MSE also has gone up from curve sections to straight sections. This could be because straight section data is spread more than the curve data as shown in Figure 6.

\section{Conclusions}

Predicting rail track degradation on time and carrying out maintenance accordingly are a plan that all the major authorities that are responsible for maintaining and managing the rail networks try to implement right across the world. The intention behind this exercise is to improve the cost efficiency by reducing the unnecessary costs related to carrying out maintenance work too early or too late. There are many types of research that have been done on developing such models for heavy rail tracks. However, there is a need for a proper model which can predict the degradation in light rail tracks. In this paper, firstly the raw data was captured by connecting the MATLAB to the database, then it is preprocessed, and the outliers are eliminated due to the messy structure of the data environment. Following that to cover all noisy environment ANFIS model was utilized; the model has five membership 
functions in the antecedent for all three inputs and is put forward to model rail track degrading using the data for Melbourne tram network between 2010 and 2015. The data is consisting of gauge values for two previous years and the MGT value. For modeling the system $70 \%$ of the data is used for the training purpose, and the remaining was used for testing the system. Results show that the model can predict the gauge values for the coming year by the $R^{2}$ value of 0.60 and 0.78 for the curves and straight sections, respectively, and the MSE error for the section are 0.7350 and 1.7335, respectively. These models could be utilized to predict the degradation for the year 2016 onwards, and maintenance work could be scheduled according to the results from degradation prediction models.

\section{Conflicts of Interest}

The authors declare that there are no conflicts of interest regarding the publication of this paper.

\section{Acknowledgments}

The authors would like to acknowledge the support and collaboration of Yarra Trams for providing this research with the required data.

\section{References}

[1] Y. Sato, "Japanese Studies on Deterioration of Ballasted Track," Vehicle System Dynamics, vol. 24, no. 1, pp. 197-208, 1995.

[2] Y. Satoh, "Experiment on Ballast Settlement due to Repeated Loading," Railway Technical Research Report, 1959.

[3] Y. Satoh, A. Onishi, and S. Tanaka, "Experiment on grading of crushed stone ballast," Railway Technical Research Report, 1961.

[4] K. Demharter, Setzungsverhalten des Gleisrostes unter vertikaler Lasteinwirkung, 1982.

[5] A. Premathilaka, S. Costello, and R. Dunn, "Development of a deterministic rail-wear prediction model," Road \& Transport Research: A Journal of Australian and New Zealand Research and Practice, vol. 19, p. 40, 2010.

[6] A. R. Andrade and P. F. Teixeira, "Statistical modelling of railway track geometry degradation using Hierarchical Bayesian models," Reliability Engineering \& System Safety, vol. 142, article no. 5318, pp. 169-183, 2015.

[7] R. G. Mishalani and S. M. Madanat, "Computation of infrastructure transition probabilities using stochastic duration models," Journal of Infrastructure Systems, vol. 8, no. 4, pp. 139-148, 2002.

[8] M. N. S. Hadi, "Neural networks applications in concrete structures," Computers \& Structures, vol. 81, no. 6, pp. 373-381, 2003.

[9] J. Sadeghi and H. Askarinejad, "Application of neural networks in evaluation of railway track quality condition," Journal of Mechanical Science and Technology, vol. 26, no. 1, pp. 113-122, 2012.

[10] H. Guler, "Prediction of railway track geometry deterioration using artificial neural networks: A case study for Turkish state railways," Structure and Infrastructure Engineering, vol. 10, no. 5, pp. 1-13, 2014.
[11] Y. Shafahi, P. Masoudi, and R. Hakhamaneshi, "Track Degradation Prediction Models, Using Markov Chain," in Proceedings of the 8th World Congress on Railway Research, Artificial Neural and Neuro-Fuzzy Network, Seoul, Korea, 2008.

[12] S. Moridpour and R. Hesami, "Degradation and performance specification of Melbourne tram tracks," in Proceedings of the 3 rd International Conference on Transportation Information and Safety, ICTIS 2015, pp. 270-276, chn, June 2015.

[13] M. Yousefikia, S. Moridpour, S. Setunge, and E. Mazloumi, "Modeling degradation of tracks for maintenance planning on a tram line," Journal of Traffic and Transportation Engineering, vol. 2, 2014.

[14] S. Moridpour, E. Mazloumi, and R. Hesami, "Application of artificial neural networks in predicting the degradation of tram tracks using maintenance data," Applied Big Data Analytics in Operations Management, 2017, IGI Global.

[15] M. Karimpour, L. Hitihamillage, N. Elkhoury, S. Moridpour, and R. Hesami, "Nonlinear estimation model for rail track deterioration," in 19th International Conference on Transportation Economics and Transportation Systems, 2017.

[16] J. S. R. Jang, "ANFIS: adaptive-network-based fuzzy inference system," IEEE Transactions on Systems, Man, and Cybernetics, vol. 23, no. 3, pp. 665-685, 1993. 


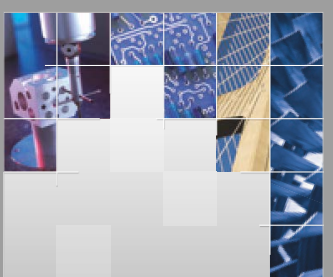

\section{Enfincering}
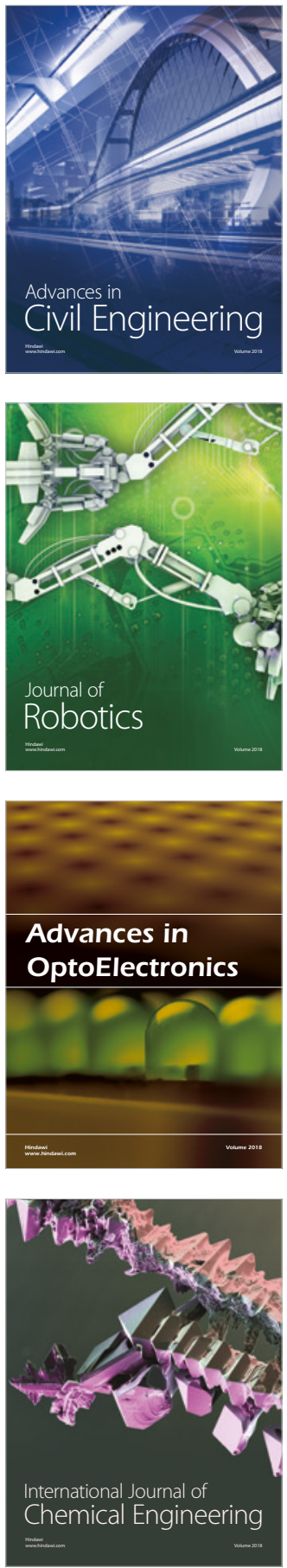

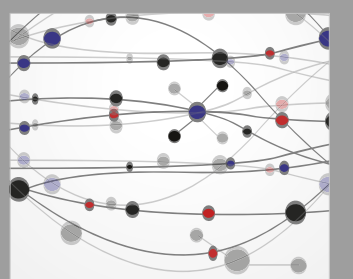

\section{Rotating \\ Machinery}

The Scientific World Journal

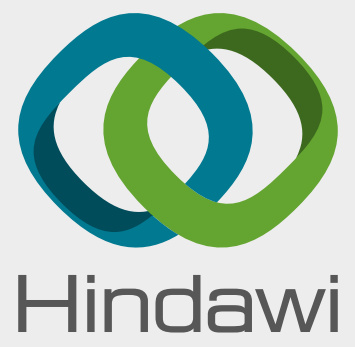

Submit your manuscripts at

www.hindawi.com
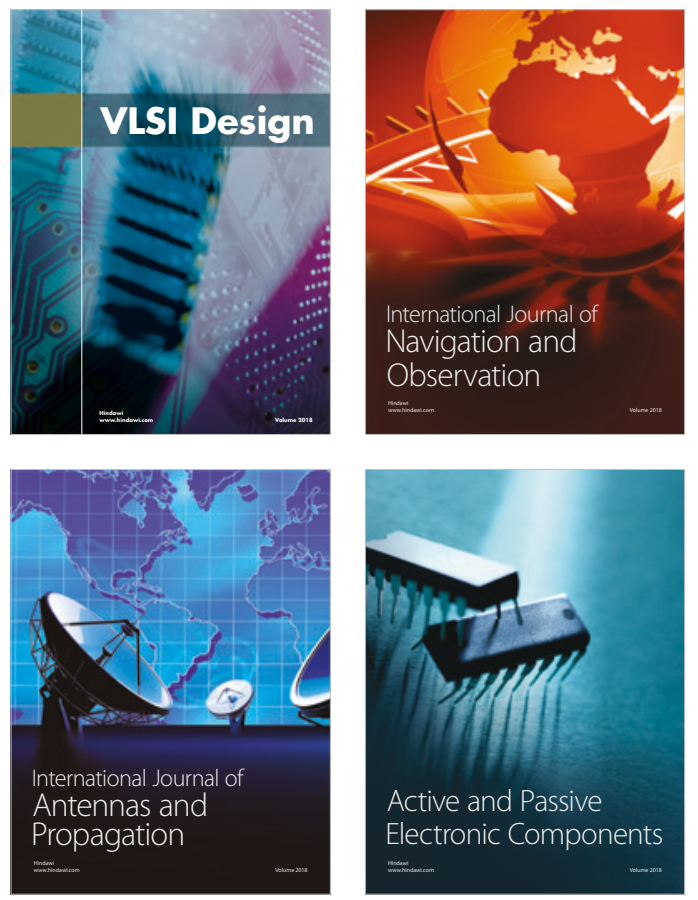
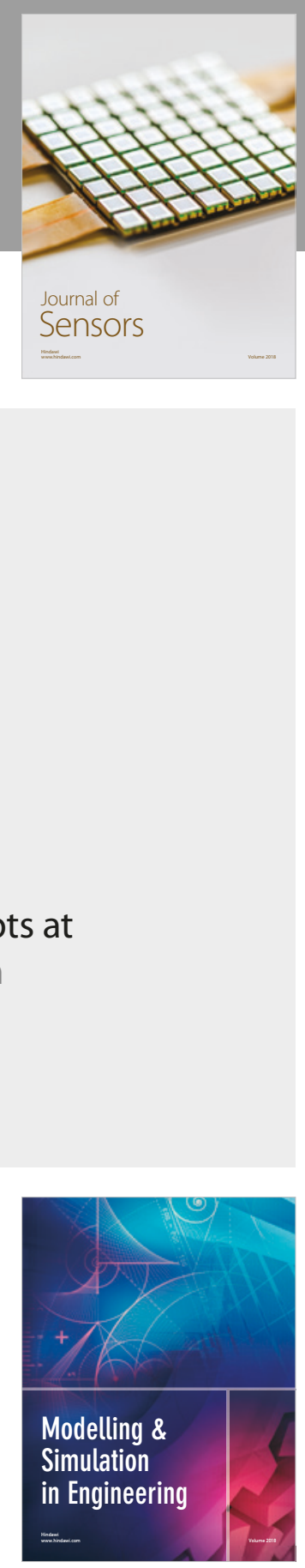

\section{Advances \\ Multimedia}
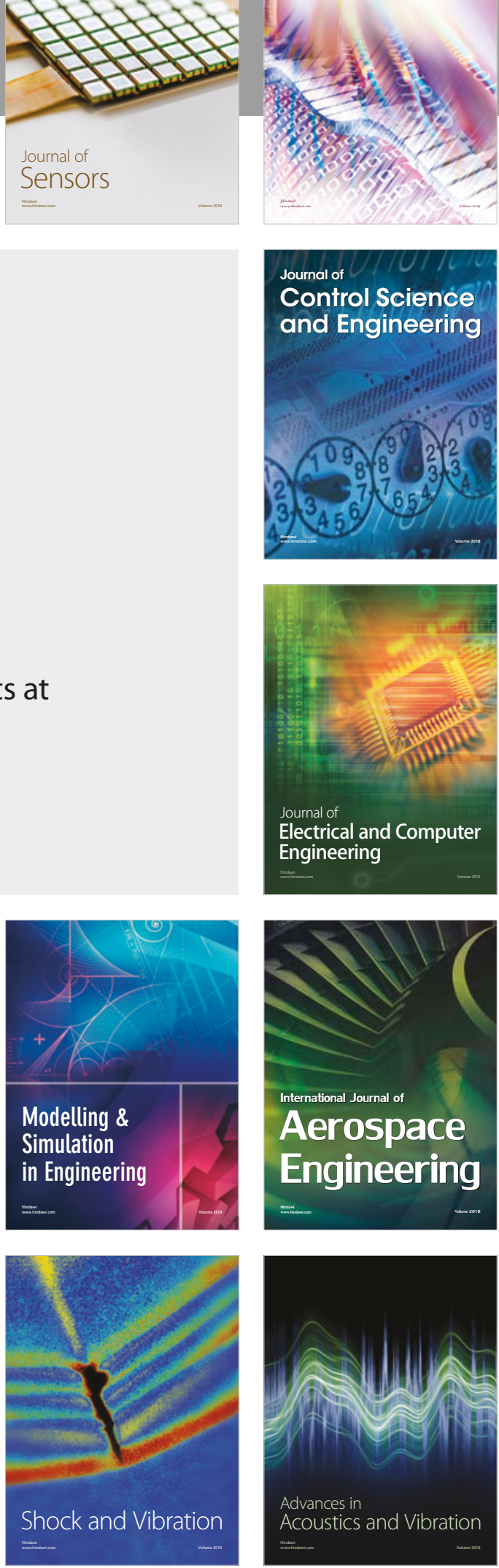